guide together with overheads that can be photocopied onto transparencies. The training guide can be used to develop understanding of needs assessment or to develop interrater reliability among research staff.

For anyone working in the field of mental health, parenting and childcare, the CAN-M will be a useful assessment tool, not just in relation to needs for clinical care, but in relation to needs for social care and support with safeguarding. Risks are clearly related to unmet needs for support with parenting and in these times of high anxiety about safeguarding, the publication of the CAN-M is very timely.

Andrew Kent St George's, University of London, Division of Mental Health, Hunter Wing, Cranmer Terrace, London SW17 ORE, UK. Email: akent@sgul.ac.uk

doi: 10.1192/bjp.bp. 108.060228

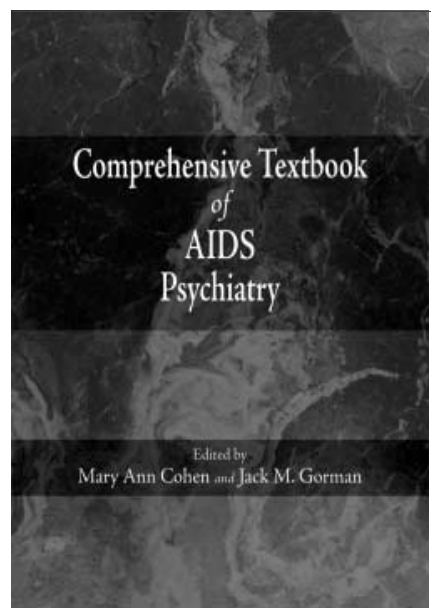

\section{Comprehensive Textbook of AIDS Psychiatry}

Edited by Mary Ann Cohen \& Jack M. Gorman. Oxford University Press. 2007 £57.00 (hb). 656pp. ISBN: 9780195304350 concerns sexual dysfunction - no review or discussion of this very common problem in people with HIV is provided beyond a passing reference to antiretroviral use.

A very detailed and comprehensive chapter on psychopharmacological interventions and drug interactions with anti-retrovirals describes the potential risks of adding psychotropic drugs to complex medical treatments. Although a first examination of the evidence might lead some psychiatrists to a prescribing paralysis, cautious use of a limited number of safe antidepressants and other psychotropics will avoid serious harmful interactions. Two emerging topics receive attention: the mental health impact of hepatitis C, both as comorbid with HIV but also in its own right, as an infection with likely neurocognitive effects, and the mental consequences of ageing with HIV infection.

Apart from the lack of attention to the mental health impact of HIV in resource-poor countries, the discussion of ethical and legal issues is limited, so that the concerns that are often raised by psychiatrists about consent for HIV testing, capacity for medical decisions and use of compulsory psychiatric treatment are addressed only briefly.

Overall, this is a good, solid book for the medical library and for general hospital mental health teams dealing with people with HIV, but possibly too detailed for the psychiatrist in training.

Jose Catalan Consultant General Hospital Psychiatrist, Department of Psychological Medicine, Chelsea and Westminster Hospital, 369 Fulham Road London SW10 9NH, UK. Email: j.catalan@imperial.ac.uk

doi: 10.1192/bjp.bp.109.066472

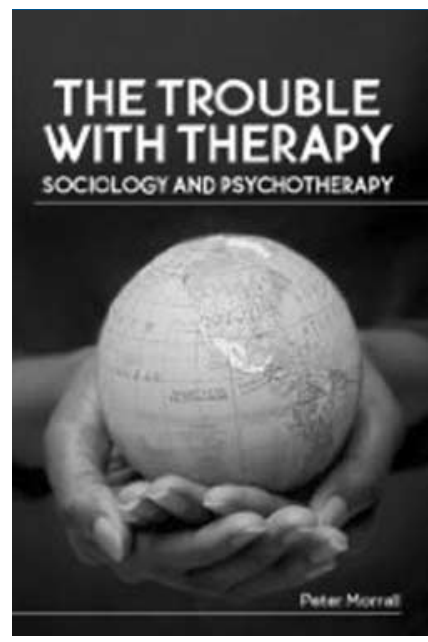

\section{The Trouble with Therapy: Sociology and Psychotherapy}

By Peter Morrall

McGraw Hill Open University Press. 2008. $£ 21.99$ (pb). 253pp.

ISBN: 9780335218752

With two forewords and divided into ten sections with a total of 41 chapters, this volume edited by two New York-based psychiatrists aims to do justice to its title. It could have been subtitled 'The view from the USA, had it not been for the fact that one of its 72 contributors is based in Canada. Among the rest, the majority work in New York and a handful in Omaha, Nebraska, in California and in Florida. Unsurprisingly in view of the origin of the contributors, the overwhelming majority of the references quoted and the topics addressed are from North America and relevant to that part of the developed world. Although the level of expertise and practical knowledge of many of the authors is a great strength of this textbook, the rather narrow geographical and social context of its scope is a limitation.

There are good, succinct chapters on the epidemiology of mental disorders in people with HIV infection, anxiety disorders and suicide ideation. Chapters on HIV-associated fatigue and insomnia cover important neglected topics in a practical and well-researched way. Discussion of the effects of HIV on brain function is detailed and exhaustive, but it would have been better not to have allocated the material to five different chapters. Inevitable repetition and overlap could have been avoided and a critical evaluation of the evidence presented in a much clearer way. Two chapters dealing with severe psychiatric disorders and risk of HIV infection and transmission will be of particular interest to general psychiatrists. Reviews of medical comorbidities with mental manifestations and interventions to increase treatment adherence are practical and clear. A surprising omission

It is now nearly 50 years since the action-research sociologist Erving Goffman turned his insider-view daily notes into a devastating critique of the realities of mental hospital life. Back then, sociologists and psychotherapists were natural allies rallying to the flag of community psychiatry, united in opposition to the hegemonic medical model. Academically, social psychiatry was the sexy place to be, with as many as seven UK units funded by the Medical Research Council charting the social and psychological impact of mental hospital closure, the social origins of depression, the epidemiology of suicide, the impact of mental illness in primary care, and the relationship between class and mental health. Today all has changed. Neuropsychiatry rules; Asylums (1961) lies unread; the few remaining sociologists are in search of new targets for radical assault. 
Psychotherapy is certainly much in need of sociological scrutiny. What is the class composition of psychotherapists and their clients? Can psychoanalysis realistically be provided for the masses away from the ivory towers of Hampstead? Does ethnicity influence choice of therapy or psychopharmacology? What, if anything, is the impact of psychotherapy as a social movement encompassing new ways of ethically and environmentally sound living? Is psychotherapy a valid profession? Does it instil creative opposition to prevailing mores or is it an instrument of subtle social control? What are we to make of the 'model wars' within psychotherapy - is the battle between psychoanalysis and cognitive-behavioral therapy a surrogate for Thatcherite assault on middle class complacency? Will registration of psychotherapists legitimise a valid social movement or represent the final bureaucratisation of radical protest? Is happiness a sensible goal for therapy or should we embrace Freud's aim of replacing neurotic misery with ordinary human suffering?

Morell's book, which has the feel of a worked-up set of firstyear sociology lectures, touches on many of these issues but, sadly, goes into none of them in depth. He himself seems to have had unfortunate experiences of therapy and describes himself as 'not just a trouble-maker [but] an angry trouble-maker'. Through this superficial analysis, interspersed with a few lists of therapy organisations and paragraph-length summaries of major sociology theorists, stalks the character of Heather, a supposed therapy seeker, and her 'barefoot' therapist Len, who first seduces and then is rejected by her. Morell seems to approve of Len in preference to trained therapists, whom he sees as peddling prostituted love under the guise of professionalism. It is all very confusing, to no one more so than the author himself.

When dealing with a problematic patient it is always a good principle to try to find something likeable about them. It was both surprising and a relief to learn from Morrall (p. 186) that if I ... were to succumb to madness it is the services of a psychiatrist that may be called upon. It is most definitely not a deconstructed sociologist or anti-psychiatrist'. This book is slightly mad. My professional advice (although of course as a psychotherapist I never give advice) is that the author retrain immediately as a neuroscientist. The jobs are there; the ground unshifting. The sociology of psychotherapy needs empirical data and subtle conceptualisation, not recycled right-on 60s clichés. The subject it seems is too tricky to be left to the sociologists, while psychiatry is beguiled by the romance of neuroscience. Oh, where are the Browns and Leffs of yesteryear?

Jeremy Holmes Department of Clinical Psychology, University of Exeter EX4 4QG, UK

doi: 10.1192/bjp.bp.109.064550 